\title{
The Influence of the Earth's Oblateness on the Energy Integral and Some Characteristics of a Spacecraft's Orbit
}

\author{
V. V. Ivashkin ${ }^{a, b}$, * \\ ${ }^{a}$ Keldysh Institute of Applied Mathematics, Russian Academy of Sciences, Moscow, Russia \\ ${ }^{b}$ Bauman Moscow State Technical University, Moscow, 105005 Russia \\ *e-mail: Ivashkin@keldysh.ru
}

Received January 13, 2021; revised February 16, 2021; accepted April 15, 2021

\begin{abstract}
Taking into account the oblateness of the Earth with the help of the second zonal harmonic of the potential of the Earth's gravitational field modifies the integral of the energy of near-Earth motion in comparison with the Keplerian model of analysis. On the basis of this integral, an explicit analytical structure of the change in the main orbital parameter-the Keplerian energy constant-is obtained. This method of taking into account the oblateness of the Earth also makes it possible to estimate the correction, in comparison with the standard unperturbed analysis, in the departure speed of a spacecraft from the near-Earth waiting orbit when flying to the Moon or another planet.
\end{abstract}

DOI: $10.1134 / \mathrm{S} 001095252105004 \mathrm{X}$

\section{FORMULATION OF THE PROBLEM}

During a spacecraft's flight or, in general, for a material point in the central Newtonian gravitational field of the Earth, the motion of a spacecraft in a nonrotating geocentric geoequatorial coordinate system satisfies the equation

$$
d^{2} \mathbf{r} / d t^{2}=\partial U_{0} / \partial \mathbf{r}^{*}=\partial(\mu / r) / \partial \mathbf{r}^{*}=-\left(\mu / r^{3}\right) \mathbf{r},
$$

where $\mathbf{r}(x, y, z)$ is the radius vector of a point $(\mathrm{SC} ; r=|\mathbf{r}|)$, * is the transposition sign, and $\mu$ is the gravitational parameter of the Earth;

$$
U_{0}(\mathbf{r})=\mu / r
$$

is potential of the Earth's gravity without taking into account the disturbances. System (1) corresponds to Keplerian motions in the gravitational field of a spherical, homogeneous Earth. One of the most important first integrals in this case is the energy integral [1-6]:

$$
V^{2}-2 U_{0}=V^{2}-2 \mu / r=h_{k}, h_{k}=-\mu / a,
$$

where $V=|\mathbf{V}|, \mathbf{V}$ being the geocentric speed of the point; $h_{k}$ is the Keplerian energy constant; and element $a$ in the case of elliptical motion when $h_{k}<0$ is the semimajor axis of the orbit. In hyperbolic motion, when $h_{k}>0, a<0$, modulus $|a|=\alpha$ [3] has a geometric sense. Let us consider how integral (3) changes when taking into account the perturbation from the oblateness of the Earth as a body of revolution, as well as its use in the analysis of the orbital motion of a material point.

\section{GENERALIZED INTEGRAL OF ENERGY TAKING INTO ACCOUNT THE OBLATENESS OF THE EARTH}

In the simplest case of analyzing motion near the Earth to take into account its oblateness in potential of the Earth $U$ to main member $U_{0}$, second zonal harmonic $U_{2}$ is added $[2,3,5,6]$ :

$$
\begin{gathered}
U(\mathbf{r})=U_{0}(\mathbf{r})+U_{2}(\mathbf{r}) ; \\
U_{2}(\mathbf{r})=-\frac{\varepsilon}{r^{3}}\left(\sin ^{2} \varphi-\frac{1}{3}\right)=-\frac{\varepsilon}{r^{3}}\left(\frac{z^{2}}{r^{2}}-\frac{1}{3}\right), \\
\varepsilon=(3 / 2) J_{2} \mu R_{\mathrm{E}}^{2},
\end{gathered}
$$

where $\varphi$ is the geocentric latitude of the spacecraft; $J_{2}=-\mathrm{C}_{20}$ is the zonal harmonic coefficient of the second order; $R_{\mathrm{E}}$ is the average equatorial radius of the Earth, $R_{\mathrm{E}} \approx 6378.137 \mathrm{~km} ; J_{2} \approx 1082.63 \times 10^{-6}$ [6]; and $\varepsilon \approx 2.63328 \times 10^{10} \mathrm{~km}^{5} / \mathrm{s}^{2}$. Equation of motion (1) changes:

$$
\mathrm{d}^{2} \mathbf{r} / \mathrm{d} t^{2}=\partial U / \partial \mathbf{r}^{*}
$$

where $U(\mathbf{r})$ is potential (4), (2), (5). To the main acceleration (1), a disturbing $\mathbf{a}_{\mathrm{P}}$, which is the gradient of the function $U_{2}$ and in rectangular coordinates it will be written in the form [2]:

$$
\begin{gathered}
a_{P x}=\frac{\varepsilon}{r^{4}}\left(5 \frac{z^{2}}{r^{2}}-1\right) \frac{x}{r} ; \quad a_{P y}=\frac{\varepsilon}{r^{4}}\left(5 \frac{z^{2}}{r^{2}}-1\right) \frac{y}{r} ; \\
a_{P z}=\frac{\varepsilon}{r^{4}}\left(5 \frac{z^{2}}{r^{2}}-3\right) \frac{z}{r} .
\end{gathered}
$$


A doubled total mechanical energy of motion of a point of unit mass $h(\mathbf{r}, \mathbf{V})$ has the form

$$
\begin{aligned}
h & =V^{2}-2 U=V^{2}-2 U_{0}-2 U_{2} \\
& =V^{2}-2 \mu / r+\frac{2 \varepsilon}{r^{3}}\left(\frac{z^{2}}{r^{2}}-\frac{1}{3}\right) .
\end{aligned}
$$

Theorem. The total mechanical energy of motion of a material point in model (6), where potential $U(\mathbf{r})$ corresponds to (4), (2), and (5), is constant on the trajectory of the point.

Indeed, in this case, the acceleration in (6) is determined by the single-valued scalar force function $U=U(\mathbf{r})$, the motion occurs in a potential field, force function $U=U(\mathbf{r})$ is a potential that does not depend on time. It then follows from the general theorem of mechanics $[1,4]$ that, on the trajectory of point $d h / d t=0$, by virtue of equation of motion (6), $h=$ const. It is possible to show this directly using (6), (1), (7). Therefore, on any trajectory of a point the energy (8) is constant, we have the first integral:

$$
h=V^{2}-\frac{2 \mu}{r}+\frac{2 \varepsilon}{r^{3}}\left(\frac{z^{2}}{r^{2}}-\frac{1}{3}\right)=h_{K}-2 U_{2}=\text { const. }
$$

We will call this integral the "generalized energy integral," bearing in mind that it generalizes integral (3) to the case of taking into account the Earth's oblateness when calculating the trajectory of a material point the SC.

Remark 1. It follows from (8), (9) that, in this model of the potential, for a given energy constant $h$, the movement of a point occurs in the area of space $h+2 U=h+2 U_{0}+2 U_{2}=h+\frac{2 \mu}{r}-\frac{2 \varepsilon}{r^{3}}\left(\frac{z^{2}}{r^{2}}-\frac{1}{3}\right)=$ $V^{2} \geq 0$. The difference from the Keplerian case is visible.

Remark 2. This approach can be applied to a more complete model of zonal harmonics. Using the model of the Earth as a body of revolution symmetric about the equatorial plane, for example, and adding a zonal harmonic of the fourth order to potential $U$ in addition to the second one, the generalized energy integral takes the form

$$
\begin{aligned}
h=V^{2} & -2 U=V^{2}-2 U_{0}-2 U_{2}-2 U_{4}=V^{2}-\frac{2 \mu}{r} \\
& +\frac{2 \varepsilon}{r^{3}}\left(\frac{z^{2}}{r^{2}}-\frac{1}{3}\right)-\frac{2 \chi}{r^{5}}\left(\frac{z^{4}}{r^{4}}-\frac{6}{7} \frac{z^{2}}{r^{2}}+\frac{3}{35}\right) .
\end{aligned}
$$

One can also use the entire expansion of the potential in zonal harmonics, which allows one to improve the accuracy and take into account the asymmetry about the equator:

$$
U=\frac{\mu}{r}\left[1-\sum_{n=2}^{\infty} J_{n}\left(\frac{R_{\mathrm{E}}}{r}\right)^{n} P_{n}(\sin \varphi)\right] .
$$

Here, $P_{n}(\sin \varphi)$ are $n$ th-order Legendre polynomials. For the Earth, the following coefficients hold after the second order: $J_{3}=-2.53 \times 10^{-6}$ and $J_{4}=-1.61 \times$ $10^{-6}$ [6], i.e., around three orders of magnitude less than $J_{2}$.

Remark 3. In this case, the potential axisymmetric force field is also the integral of the axial angular momentum of the point [4]: $\mathbf{M}_{\mathrm{z}}=\left(\mathbf{e}_{\mathrm{z}},[\mathbf{r}, \mathbf{V}]\right)=\mathrm{m}=$ const, where $\mathbf{e}_{\mathrm{z}}$ is the ort along the axis of rotation of the Earth.

\section{CHANGE IN THE KEPLERIAN ENERGY CONSTANT ON ORBITS OF DEPARTURE TO THE MOON AND PLANETS}

Having written out integral (9) for initial point of the trajectory $\mathbf{x}_{0}\left(\mathbf{r}_{0}, \mathbf{V}_{0}, t_{0}\right)$ and for some other point $\mathbf{x}_{\mathrm{f}}$ $\left(\mathbf{r}_{\mathrm{f}}, \mathbf{V}_{\mathrm{f}}, t_{\mathrm{f}}\right)$, we obtain the ratio

$$
\begin{aligned}
& h=V_{0}^{2}-\frac{2 \mu}{r_{0}}+\frac{2 \varepsilon}{r_{0}^{3}}\left(\frac{z_{0}^{2}}{r_{0}^{2}}-\frac{1}{3}\right) \\
& =V_{f}^{2}-\frac{2 \mu}{r_{f}}+\frac{2 \varepsilon}{r_{f}^{3}}\left(\frac{z_{f}^{2}}{r_{f}^{2}}-\frac{1}{3}\right) .
\end{aligned}
$$

Let us apply it to the analysis of the spacecraft departure trajectories from the Earth to the Moon and planets.

It follows from (10) that the change in the Keplerian energy constants in accurate analysis satisfies the relationship

$$
\begin{aligned}
\Delta h_{k}=h_{k f}-h_{k 0} & =2 U_{2 f}-2 U_{20}=-\frac{2 \varepsilon}{r_{f}^{3}}\left(\frac{z_{f}^{2}}{r_{f}^{2}}-\frac{1}{3}\right) \\
& +\frac{2 \varepsilon}{r_{0}^{3}}\left(\frac{z_{0}^{2}}{r_{0}^{2}}-\frac{1}{3}\right), \\
z / r=\sin \varphi= & \sin i \sin u=\sin i \sin (\omega+\theta),
\end{aligned}
$$

where $i$ and $\omega$ are the inclination and argument of the orbit perigee and $u$ and $\theta$ are the latitude argument and true point anomaly.

Remark 4. Energy integral (9) and exact relation (11) for the change in $\Delta h_{k}$ are valid for any orbit-in particular, for any values of inclination and eccentricity.

If we proceed from Keplerian constant $h_{k}$ to semiaxis $a$ (3) and linearize according to $a$, then we obtain variation $\Delta a$ as a first approximation from (11):

$$
\Delta a \approx\left(a_{0}^{2} / \mu\right) \Delta h_{k} \text {. }
$$

During the spacecraft's flight to the planet, the departure from the Earth will occur in a hyperbolic orbit (in an osculating approximation). For purposes of assessment, we take the velocity "at infinity" for this orbit as $V_{\infty}=3-4 \mathrm{~km} / \mathrm{s}$. In this case, distance $r_{f}$ increases without bound and the penultimate term in (11), $2 U_{2 f}$, 
tends to zero; therefore, the change in Keplerian energy constants $\Delta h_{K}$ tends to limiting value $\Delta h_{K l}$.

$$
\Delta h_{K} \rightarrow \Delta h_{K l}=-2 U_{20}=\frac{2 \varepsilon}{r_{0}^{3}}\left(\sin ^{2} \varphi_{0}-\frac{1}{3}\right) .
$$

If $\sin ^{2} \varphi_{0}<\frac{1}{3}\left(\left|\varphi_{0}\right|<\varphi_{0}^{*}=35.2644^{\circ}\right)$, then $\Delta h_{K l}<0$. If $\sin ^{2} \varphi_{0}>\frac{1}{3}$, then $\Delta h_{K l}>0$. For a flight to the Moon, we assume that a highly elongated near-parabolic elliptical orbit has a perigee at starting point $r_{\pi}=r_{0} \approx$ $6578 \mathrm{~km}$; initial distance at apogee $r_{\alpha}$ corresponds to distance to the Moon $r_{M}$ when the spacecraft approaches the Moon; $r_{\alpha} \geq\left(r_{M}-\Delta r_{\alpha}\right) ; r_{M} \approx 360000-$ $405000 \mathrm{~km}$; and $\Delta r_{\alpha}<0$ is the correction for decreasing $r_{\alpha}$ due to the oblateness of the Earth, $\Delta r_{\alpha} \approx 2 \Delta a$. In (11), distance $r_{f}$ increases in the process of spacecraft motion from $r_{0}$ to $r_{M}<\infty$, while the penultimate term in (11) decreases to a small value $\left(\sim 10^{-6} \mathrm{~km}^{2} / \mathrm{s}^{2}\right)$ and the limiting change in Keplerian constant $h_{K l}$ is

$$
\Delta h_{K l} \approx-2 U_{20}=\frac{2 \varepsilon}{r_{0}^{3}}\left(\sin ^{2} \varphi_{0}-\frac{1}{3}\right) .
$$

For numerical estimates, for the initial point of departure to the Moon and planets from the nearEarth reference orbit, we take, for definiteness, $u_{0}=0$, which is close to the characteristics of interplanetary and lunar flights. In formulas (13), (14), then, $\varphi_{0}=0$ and $z_{0}=0$, and we obtain

$$
\Delta h_{K l} \approx-2 \varepsilon /\left(3 r_{0}^{3}\right) .
$$

In this case, for departures from the Earth both to the planet in a hyperbolic orbit and to the Moon in an elongated elliptical orbit, $\Delta h_{K l} \approx-0.0617 \mathrm{~km}^{2} / \mathrm{s}^{2}$. Numerical calculations have confirmed these estimates [7, 8]. When $h_{K}<0$, this change in the Keplerian energy constant (14) corresponds to a change in the semimajor axis of the orbit, in accordance with (12): $\Delta a \approx-6200 \mathrm{~km}$ at $a_{0}=200000 \mathrm{~km}, \Delta a \approx-8900 \mathrm{~km}$ at $a_{0}=220000 \mathrm{~km}$, and $\Delta a \approx-56000 \mathrm{~km}$ at $a_{0}=600000 \mathrm{~km}$. In practice, this change occurs quickly, during the first $3 \mathrm{~h}$ or so of the spacecraft's initial flight from the Earth, with an increase in distance $r_{f}$ up to $\sim 70000 \mathrm{~km}$.

\section{DEPARTURE SPEED OF THE SPACECRAFT FROM THE NEAR-EARTH REFERENCE ORBIT}

The change in the Keplerian energy constants caused by the oblateness of the Earth, within the framework of model (4), (5), leads to a change in the initial velocity of the spacecraft's departure from the Earth in comparison with the Keplerian model of motion. For the analysis to be correct, let us set a certain value of the Keplerian energy constant for departure orbit $h_{K g}\left(\right.$ or $\left.V_{\infty}, a\right)$. Then, in the Keplerian model of spacecraft motion (1), (2), by virtue of integral (3), the initial velocity is

$$
V_{0}=\left(h_{K g}+2 \mu / r_{0}\right)^{1 / 2} .
$$

Taking into account the oblateness of the Earth, in model (4), (5), under the condition $h_{K f}=h_{K g}$, by virtue of integral (10), the initial velocity is determined from the relation

$$
V_{0}=\sqrt{h_{K g}+\frac{2 \mu}{r_{0}}+2 U_{20}-2 U_{2 f}}=\sqrt{h_{K g}+\frac{2 \mu}{r_{0}}+\Delta h},
$$

where

$$
\begin{gathered}
\Delta h=2 U_{20}-2 U_{2 f}=\frac{2 \varepsilon}{r_{f}^{3}}\left(\frac{z_{f}^{2}}{r_{f}^{2}}-\frac{1}{3}\right) \\
-\frac{2 \varepsilon}{r_{0}^{3}}\left(\frac{z_{0}^{2}}{r_{0}^{2}}-\frac{1}{3}\right) \approx-\frac{2 \varepsilon}{r_{0}^{3}}\left(\frac{z_{0}^{2}}{r_{0}^{2}}-\frac{1}{3}\right) .
\end{gathered}
$$

Let us provide numerical estimates for the case $z_{0}=0$. When flying from the Earth to a planet in a geocentric orbit with $V_{\infty}=3-4 \mathrm{~km} / \mathrm{s}$, taking into account the Earth's oblateness in (17), increases initial velocity $V_{0}$ in comparison with Keplerian case (16) from 11.41011.713 to $\sim 11.413-11.716 \mathrm{~km} / \mathrm{s}$, i.e., by $\sim 3 \mathrm{~m} / \mathrm{s}$. When flying to the Moon, when $r_{\alpha}=r_{M}+\left|\Delta r_{\alpha}\right|, r_{M}=400000 \mathrm{~km}$, taking into account oblateness increases initial speed $V_{0}$ from $\sim 10.919$ to $\sim 10.922 \mathrm{~km} / \mathrm{s}$, i.e., also by $\sim 3 \mathrm{~m} / \mathrm{s}$. This leads to an increased initial osculating semimajor axis of the orbit relative to the Keplerian case by $\sim 6500 \mathrm{~km}$, while initial apogee distance $r_{\alpha}$ is increased by $\sim 13000 \mathrm{~km}[7,8]$ as noted above.

\section{ESTIMATION OF THE CHANGE \\ IN THE KEPLERIAN ENERGY CONSTANT IN THE FIRST APPROXIMATION}

When analyzing the spacecraft's motion in a hyperbolic and highly elongated elliptical orbit, we used the distance to the center of the Earth as a parameter on the trajectory. In the general case, it is more convenient to use true anomaly $\theta$ [9], time $t$, or average anomaly $M[10,11]$. We transform relations (11), (12) to use angle $\theta$ when determining the change in Keplerian constant $\Delta h_{K}$ (or semiaxis $\Delta a$ ) as a first approximation. Then, according to (11), (11a) and taking into account the orbital equation, we obtain

$$
\begin{gathered}
\Delta h_{k}=2 U_{2 f}-2 U_{20} \approx-\frac{2 \varepsilon}{p^{3}}(1+e \cos \theta)^{3} \\
\times\left[\sin ^{2} i \sin ^{2}(\omega+\theta)-\frac{1}{3}\right]-2 U_{20},
\end{gathered}
$$

where the orbital elements are focal parameter $p$, eccentricity $e, i$, and $\omega$, which are equal to their initial values $p_{0}, e_{0}, i_{0}$, and $\omega_{0}$. Change $\Delta a$ is determined by (12). If the orbit is elliptical, $a>0$, and the multiturn motion of the point is considered, then the motion can be aver- 
aged. To simplify this procedure, using elementary identical trigonometric transformations, we lead $2 U_{2 f}$ to the sum of constant $c_{0 \theta}$ and several terms of the form $\left(2 \varepsilon / p^{3}\right) c_{j}(i, e) \cos \left(n_{j} \omega+m_{j} \theta\right)$, where $n_{j}$ and $m_{j}$ are whole numbers:

$$
\begin{gathered}
2 U_{2 f}=c_{0 \theta}+\left(2 \varepsilon / p^{3}\right) \sum_{j=1}^{J} c_{j}(i, e) \cos \left(n_{j} \omega+m_{j} \theta\right), \\
c_{0 \theta}=\left(2 \varepsilon / 3 p^{3}\right)\left(1-\frac{3}{2} \sin ^{2} i\right)\left(1+\frac{3}{2} e^{2}\right) .
\end{gathered}
$$

This constant gives the "average" shift of Keplerian energy constant $h_{K}$ due to the oblateness of the Earth according to (17). In this problem, parameter $\theta$ is convenient, but it is customary to do averaging over mean anomaly $M$. Then, after some transformations, we obtain the function value $2 U_{2 f}$ that is "average" over $M$ :

$$
c_{0 \mathrm{M}}=\left(2 \varepsilon / 3 a p^{2}\right)\left(1-\frac{3}{2} \sin ^{2} i\right)\left(1-e^{2}\right)^{1 / 2} .
$$

Remark 5. Let the celestial body not fly away from the Earth, but approach it, entering its atmosphere. The change in the Keplerian energy constant with time then occurs in the opposite direction. Moreover, distance from the Earth $r$ decreases with time and the value of potential $U_{2}$ increases to the final value corresponding to the entry into the atmosphere, $r \sim 6478 \mathrm{~km}$.

Remark 6. If a celestial body (spacecraft, asteroid, comet) approaches the Earth, moving at a great distance $(r \sim 300000-400000 \mathrm{~km})$ from the Earth in an elliptical near-parabolic orbit for which $-\Delta h_{K}<h_{K}<0$, then, due to the oblateness of the Earth, the Keplerian energy constant can increase to a positive value and this body will approach the Earth in a hyperbolic orbit. Another case is possible in which, under the influence of the Earth's oblateness, the orbit changes its structure from hyperbolic to elliptical.

\section{CONCLUSIONS}

A simplified analysis of the effect of the oblateness of the Earth as a body of revolution-based on the zonal harmonics of the gravitational potential-makes it possible to use the energy integral, which generalizes the energy integral in the Keplerian case. This makes it possible to consider some of the qualitative features of a spacecraft's motion during flight to the Moon and plan- ets and during the return to the Earth, as well as celestial bodies, asteroids, and comets closely approaching the Earth. Oblateness of the Earth can cause a change in the structure of the orbits of these bodies-from elliptical to hyperbolic and vice versa.

\section{ACKNOWLEDGMENTS}

The author expresses his sincere gratitude to V.V. Sazonov and A.A. Sukhanov for an interesting discussion of the work.

\section{REFERENCES}

1. Suslov, G.K., Teoreticheskaya mekhanika (Theoretical Mechanics), Moscow: Gostekhizdat, 1944.

2. Akim, E.L. and Eneev, T.M., Determination of spacecraft motion parameters using data of trajectory measurements, Kosmich. Issled., 1963, vol. 1, no. 1, pp. 5-50.

3. El'yasberg, P.E., Vvedenie v teoriyu poleta iskusstvennykh sputnikov Zemli (Introduction to the Theory of Flight of Artificial Earth Satellites), Moscow: Nauka, 1965.

4. Arnol'd, V.I., Matematicheskie metody klassicheskoi mekhaniki (Mathematical Methods of Classical Mechanics), Moscow: Nauka, 1979.

5. Okhotsimskii, D.E. and Sikharulidze, Yu.G., Osnovy mekhaniki kosmicheskogo poleta (Fundamentals of Space Flight Mechanics), Moscow: Nauka, 1990.

6. Chobotov, V.A., Orbital Mechanics, AIAA Education Series, Reston, VA: AIAA, 2002.

7. Ivashkin, V.V., Optimizatsiya kosmicheskikh manevrov pri ogranicheniyakh na rasstoyaniya do planet (Optimization of Space Maneuvers with Limitations on Distances to Planets), Moscow: Nauka, 1975.

8. Ivashkin, V.V., Optimal trajectories of spacecraft flight to the Moon in the Earth-Moon-Sun system, Preprint of Keldysh Inst. of Applied Mathematics, Russ. Acad. Sci., Moscow, 2001, no. 85.

9. Krause, H.G.L., Die säkularen und periodischen Störungen der Bahn eines künstlichen Erdsatteliten, in Proc. 7th International Astronautical Congress, Rome, 1956, pp. 523-585.

10. Proskurin, V.F. and Batrakov, Yu.V., Disturbances in the motion of artificial satellites caused by the compression of the Earth, Byull. Inst. Teor. Astron., 1960, vol. 7, no. 7(90), pp. 537-549.

11. Abalakin, V.K., et al., Spravochnoye rukovodstvo po nebesnoy mekhanike $i$ astrodinamike (Reference Guide to Celestial Mechanics and Astrodynamics), Moscow: Nauka, 1976. 